University of Wollongong

Research Online

Faculty of Engineering and Information

Faculty of Engineering and Information

Sciences - Papers: Part A

Sciences

2009

Solution of inverse kinematic problem for serial robot using quaterninons

Emre Sariyildiz

University of Wollongong, emre@uow.edu.au

Hakan Temeltas

Istanbul Technical University

Follow this and additional works at: https://ro.uow.edu.au/eispapers

Part of the Engineering Commons, and the Science and Technology Studies Commons

Research Online is the open access institutional repository for the University of Wollongong. For further information contact the UOW Library: research-pubs@uow.edu.au 


\title{
Solution of inverse kinematic problem for serial robot using quaterninons
}

\author{
Abstract \\ A new inverse kinematic solution for serial robot manipulators is represented in this paper. Major aims of \\ this paper are to obtain singularity avoiding inverse kinematic solutions and formulize kinematic \\ problems in a compact closed form. Our solution method is based on screw theory and it uses \\ quaternions as a screw motion operator. Screw theory methods based on line transformation. All screw \\ motions are represented as a rotation about a line together with a translation along the line with respect \\ to base frame. Thus screw theory methods do not suffer from singularities. Two quaterninos are used to \\ represent screw motion. First one is for orientation and second one is for translation. Thus we formulize \\ kinematic problems in a compact closed form. 6R-DOF industrial robot manipulators forward and inverse \\ kinematic equations are derived using this new formulation and also it compared with D-H convention \\ that is the most common method in robot kinematic.

\section{Disciplines} \\ Engineering I Science and Technology Studies

\section{Publication Details} \\ Sariyildiz, E. \& Temeltas, H. (2009). Solution of inverse kinematic problem for serial robot using \\ quaterninons. 2009 IEEE International Conference on Mechatronics and Automation, ICMA 2009 (pp. \\ 26-31). United States: IEEE.
}




\section{Solution of Inverse Kinematic Problem for Serial Robot Using Quaterninons}

\author{
Emre Sariyildiz \\ Department of Control Engineering \\ Istanbul Technical University \\ 34469 Istanbul Turkey \\ esariyildiz@itu.edu.tr
}

\author{
Hakan Temeltas \\ Department of Control Engineering \\ Istanbul Technical University \\ 34469 Istanbul Turkey \\ hakan.temeltas@itu.edu.tr
}

\begin{abstract}
A new inverse kinematic solution for serial robot manipulators is represented in this paper. Major aims of this paper are to obtain singularity avoiding inverse kinematic solutions and formulize kinematic problems in a compact closed form. Our solution method is based on screw theory and it uses quaternions as a screw motion operator. Screw theory methods based on line transformation. All screw motions are represented as a rotation about a line together with a translation along the line with respect to base frame. Thus screw theory methods do not suffer from singularities. Two quaterninos are used to represent screw motion. First one is for orientation and second one is for translation. Thus we formulize kinematic problems in a compact closed form. 6R-DOF industrial robot manipulators forward and inverse kinematic equations are derived using this new formulation and also it compared with D-H convention that is the most common method in robot kinematic.
\end{abstract}

Index Terms - Inverse Kinematic; Line Transformation; Quaternion; Screw Theory; Serial Robot;

\section{INTRODUCTION}

A general robot kinematic problem is the study of the motion of robots without considering forces and torques. The general robot kinematic problem can be separated into two sub-problems. First one is forward kinematic problem, which is to determine the position and orientation of the end effector given the values for the joint variables of the robot. The second one is inverse kinematic problem is to determine the values of the joint variables given the end effector's position and orientation. Inverse kinematic problem is more complicated than forward kinematic problem in serial robot manipulators. [1] Robot kinematic is an extensively researched subject. There are several well developed analysis techniques. There are two main approaches in robot kinematic. First one is point transformation and the second one is line transformation. However, most of existing methods are based on point transformation, lines are more fundamental to velocity analysis and hence line transformations are believed to be better suited for the kinematic and static analysis of manipulators. [2]

The most common method is Denavit and Hartenberg notation for definition of special mechanism [3]. This method is based on point transformation approach and it is used $4 \times 4$ homogeneous transformation matrix which is introduced by
Maxwell [4]. The coordinate systems are described with respect to previous one. For the base point an arbitrary base coordinate system can be used. Hence some singularity problems may occur because of this description of the coordinate systems. And also in this method 16 parameters are used to represent the transformation of rigid body while just 6 parameters are needed.

Another main method in robot kinematic is screw theory which is based on line transformations approach. The elements of screw theory can be traced to the work of Chasles and Poinsot in the early 1800s. Using the theorems of Chasles and Poinsot as a starting point, Robert S. Ball developed a complete theory of screws which he published in 1900. [5] In screw theory every transformation of a rigid body or a coordinate system with respect to a reference coordinate system can be expressed by a screw displacement, which is a translation by along a $\lambda$ axis with a rotation by a $\theta$ angle about the same axis [5]. Screw theory has two main advantages. First is that it allows a global description of rigid body motion that does not suffer from singularities due to the use of local coordinates. The second advantage is that the screw theory provides a geometric description of rigid motion which greatly simplifies the analysis of mechanisms [6].

Several operators can be used in screw theory. However, quaternion is the best operator to describe screw motion. In 1843 , the Irish mathematician W. R. Hamilton invented quaternions in order to extend three-dimensional vector algebra for inclusion of multiplications and divisions [7]. However, quaternions have had a revival in the late 20th century, primarily due to their utility in describing spatial rotations. Representations of rotations by quaternions are more compact and faster to compute than representations by matrices [8] [9]. Thus quaternion became popular in kinematic researches. Among these researches, Yang and Freudenstein are the first to apply line transformation operator mechanism by using the quaternion as the transformation operator (1964) [10]; a comparison of the computational efficiency between homogeneous transformation and quatemions is presented by Funda and Paul (1988) [8] [9]; differential equations are given by Chou (1992) [11]; a general quaternion transformation representation for robotic application is presented by Guo, Chen and Hung (1986) [12].

In this paper we present a new formulation method to solve kinematic problem of serial robot manipulators. Our method is 
based on screw theory and quaternion is used as a transformation operator. Quaternions aren't transformed into matrices to obtain forward and inverse kinematic solutions. Thus in our method, solutions are computed faster and formulizations are more compact than representation by matrices. Another important advantage of this method is that inverse kinematic solutions aren't suffered from singularities. We can reach all workspace by using this method. We need just two coordinate frames which are at the base and at the end effectors. For the other joints just axis are used to describe joint motions. All joint axis and end effectors coordinate are transformed with respect to base coordinate. Hence we can avoid singularity problem. Its geometric meaning is obvious and it is very easy to implement to the robot manipulators. 6RDOF robot manipulator is solved for forward and inverse kinematic problem and also simulation results are given.

\section{LINE GEOMETRY}

A line can be completely defined by the ordered set of two vectors. First one is point vector $(\boldsymbol{p})$ which indicates the position of an arbitrary point on line, and the other vector is free direction vector $(d)$ which gives the line direction. A line can be expressed as

\section{$L(\boldsymbol{p}, \boldsymbol{d})$}

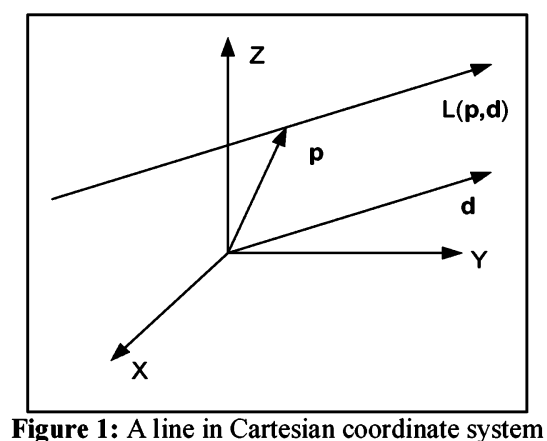

The representation $L(\boldsymbol{p}, \boldsymbol{d})$ is not minimal, because it uses six parameters for only four degrees of freedom. With respect to a world reference frame, the line's coordinates are given by a six-vector. [13]

\section{QUATERNION}

Quaternions are hyper-complex numbers of rank 4, constituting a four dimensional vector space over the field of real numbers [12] [14]. A quaternion can be represented as

$q=\left(q_{0}, q_{v}\right)$

where $q_{0}$ is a scalar and $\boldsymbol{q}_{v}=\left(q_{1}, q_{2}, q_{3}\right)$ is a vector. A quaternion with $\mathbf{q}_{\mathbf{v}}=0$, is called as a real quaternion, and a quaternion with $q_{0}=0$, is called as a pure quaternion (or vector quaternion). Addition of two quaternions is simple and it can be expressed as

$$
q_{a}+q_{b}=\left(q_{a 0}+q_{b 0}\right),\left(\boldsymbol{q}_{a v}+\boldsymbol{q}_{b v}\right)
$$

Multiplication of two quaternions is harder than addition and it can be expressed as

$q_{a} \otimes q_{b}=q_{a 0} q_{b 0}-\boldsymbol{q}_{a v} \boldsymbol{q}_{b v}, q_{a 0} \boldsymbol{q}_{b v}+q_{b 0} \boldsymbol{q}_{a v}+\boldsymbol{q}_{a v} \times \boldsymbol{q}_{b v}$ (4)

where " $\otimes "$, ,.", " $\times$ " denotes quaternion product, dot product and cross product respectively. The quaternion addition is associative and commutative. The quaternion multiplication is associative, and distributes over addition but it is not commutative.

Conjugate of the quaternion can be expressed as:

$q^{*}=\left(q_{0},-q_{v}\right)=\left(q_{0},-q_{1}-, q_{2},-q_{3}\right)$

The above equation allows us to define the quaternion norm $\|\mathrm{q}\|$ as:

$\|q\|^{2}=q \otimes q^{*}=q_{0}^{2}+q_{1}^{2}+q_{2}^{2}+q_{3}^{2}$

When $\|q\|^{2}=1$, we get a unit quaternion. Any quaternion (q) can be normalized by dividing by its norm, to obtain a unit quaternion.

The inverse of a quaternion can be expressed as:

$q^{-1}=\frac{1}{\|q\|^{2}} q^{*}$ and $\|q\| \neq 0$

that satisfies the relation $q^{-1} \otimes q=q \otimes q^{-1}=1$

For a unit-quaternion we have

$q^{-1}=q^{*}$

Unit quaternion can be defined as a rotation operator. Rotation about a unit axis $\boldsymbol{d}$ with an angle $\theta$ is defined by

$q=\left(\cos \left(\frac{\theta}{2}\right), \sin \left(\frac{\theta}{2}\right) d\right)$

\section{LINE TRANSFORMATION USING QUATERNION}

A general rigid transformation has 6 DOF. $3 \mathrm{DOF}$ is for orientation and $3 \mathrm{DOF}$ is for translation. Hence we need a transformation operator which has at least six parameters. A unit-quaternion can be used as a rotation operator. A point $\boldsymbol{p}_{\boldsymbol{b}}$ can be transformed to a point $\boldsymbol{p}_{\boldsymbol{a}}$ by using unit quaternions as follow:

$p_{a}=q \otimes p_{b} \otimes q^{*}$

where $q$ is unit-quaternion and $p_{a}$ is pure quaternion. Unitquaternions can be used for transformation of a point but general rigid transformation can't be implemented by using unit-quaternions [12]. Therefore we will use another quaternion to implement translation. Hence we have a new transformation operator which has 8 rank. General rigid transformation can be represented by using this new 
transformation operator. This transformation is very similar with pure rotation; however, not for a point but for a line.

\section{SCREW THEORY WITH QUATERNION}

\section{A. Screw Theory}

The elements of screw theory can be traced to the work of Chasles and Poinsot in the early 1800s [5]. According to Chasles all proper rigid body motions in 3-dimensional space, with the exception of pure translation, are equivalent to a screw motion, that is, a rotation about a line together with a translation along the line [5] [15]. If the line passes through the origin, we can write the screw motion as follow

$$
\left[\begin{array}{cc}
R(\theta, d) & \left(\frac{\theta}{2 \pi} p d\right) \\
0 & 1
\end{array}\right]
$$

Here $R(\theta, d)$ represents $3 \times 3$ rotation matrix about an axis in the direction of the unit vector $\boldsymbol{d}$ and through an angle $\theta$. The number $p$ is called the pitch of the screw; it is the distance moved along the axis for a complete turn about the axis. If the axis of the screw motion does not pass through origin as shown in figure 2 , a general screw motion can be written as

$$
\begin{aligned}
& T=\left[\begin{array}{cc}
I_{3 \times 3} & p \\
0 & 1
\end{array}\right]\left[\begin{array}{cc}
R(\theta, d) & \left(\frac{\theta}{2 \pi} p d\right) \\
0 & 1
\end{array}\right]\left[\begin{array}{cc}
I_{3 \times 3} & -p \\
0 & 1
\end{array}\right] \\
& T=\left[\begin{array}{cc}
R(\theta, d) & \frac{\theta}{2 \pi} p d+\left(I_{3 \times 3}-R(\theta, d)\right) d \\
0 & 1
\end{array}\right]
\end{aligned}
$$

For prismatic joints screw motion can be expressed as

$$
\left[\begin{array}{cc}
I_{3 \times 3} & \left(\frac{\theta}{2 \pi} p d\right) \\
0 & 1
\end{array}\right]
$$

For revolute joints, screw motion can be expressed as

$$
\left[\begin{array}{cc}
R(\theta, d) & \left(I_{3 \times 3}-R(\theta, d)\right) d \\
0 & 1
\end{array}\right]
$$

\section{B. Screw Motion with Quaternion}

In equation (13), screw motion is expressed by using $4 \times 4$ transformation matrices. It uses sixteen parameters while just 6 parameters are needed. Screw motion can be expressed in a more compact form by using quaternion. If we separate general screw motion as a rotation and translation, we have

Rotation: $R(\theta, d)$

Translation: $t=\frac{\theta}{2 \pi} p d+\left(I_{3 \times 3}-R(\theta, d)\right) p$

It can be expressed by using quaternion as follow:

Rotation: $q=\left(\cos \left(\frac{\theta}{2}\right), \sin \left(\frac{\theta}{2}\right) d\right)$
Translation: $t=\dot{q}+p-q \otimes p \otimes q^{*}$

where $\dot{q}$ is the amount of translation and $p$ is the position vector of some point on the line in pure quaternion form.

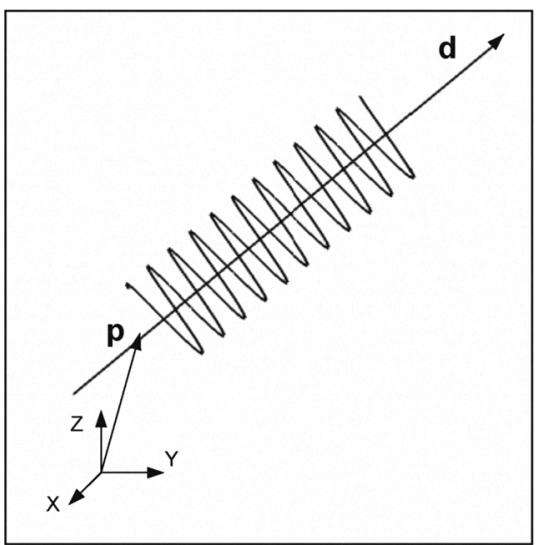

Figure 2: A general screw motion

VI. MANIPUlatoR KINEMATIC

\section{A. Forward Kinematic}

To find forward kinematics of serial robot manipulator we followed these steps:

Notation:

1. Label the joints and the links: Joints are numbered from number 1 to $n$, starting at the base, and the links are numbered from number 0 to $n$. The joints connect link i-1to link i.

2. Configuration of joint spaces: For revolute joint we describe rotational motion about an axis and we measure all joint angles by using a right-handed coordinate system. For prismatic joint we describe a linear displacement along the direction of the axis.

3. Attaching coordinate frames (Base and Tool Frames): Two coordinate frames are needed for $\mathrm{n}$ degree of freedom open-chain robot manipulator. The base frame can be attached arbitrary but in general it is attached directly to link 0 and the tool frame is attached to the end effector of robot manipulator. This notation is given for 6-DOF serial robot manipulator in figure 3 .

\section{Formulization:}

1. Determining the joint axis vector: First we attach an axis vector which describes the motion of the joint.

2. Obtaining transformation operator: For all joints we obtain quaternions for transformation operator as follow:

Prismatic Joints:

Rotation: $\quad q_{i}=(1,0,0,0)$

Translation: $q_{i}^{o}=\left(0, q_{1}, q_{2}, q_{3}\right)$ 
where $q_{i}^{o}$ is pure quaternion which indicates the amount of translation.

Revolute Joints:

Rotation: $q_{i}=\left(\cos \left(\frac{\theta_{i}}{2}\right), \sin \left(\frac{\theta_{i}}{2}\right) d_{i}\right)$

Translation: $q_{i}^{o}=p_{i}-q_{i} \otimes p_{i} \otimes q_{i}^{*}$

where $p_{i}$ is an arbitrary point on the i.th axis.

3. Formulization of rigid motion: Using (18) and (19) transformation of serial robot manipulator can be given by

$\hat{q}_{n}=q_{1} \otimes q_{2} \otimes \ldots . q_{n}$

$\hat{q}_{n}^{o}=\hat{q}_{n-1} \otimes p_{n} \otimes \hat{q}_{n-1}^{*}-\hat{q}_{n} \otimes p_{n} \otimes \hat{q}_{n}^{*}+\hat{q}_{n-1}^{o}$

where $\hat{q}_{n}$ and $\hat{q}_{n}^{o}$ indicate rotation and translation respectively. The position of the end effector can be given by

$$
\hat{q}_{e p}=\hat{q}_{n} \otimes p_{e p} \otimes \hat{q}_{n}^{*}+\hat{q}_{n}^{o}
$$

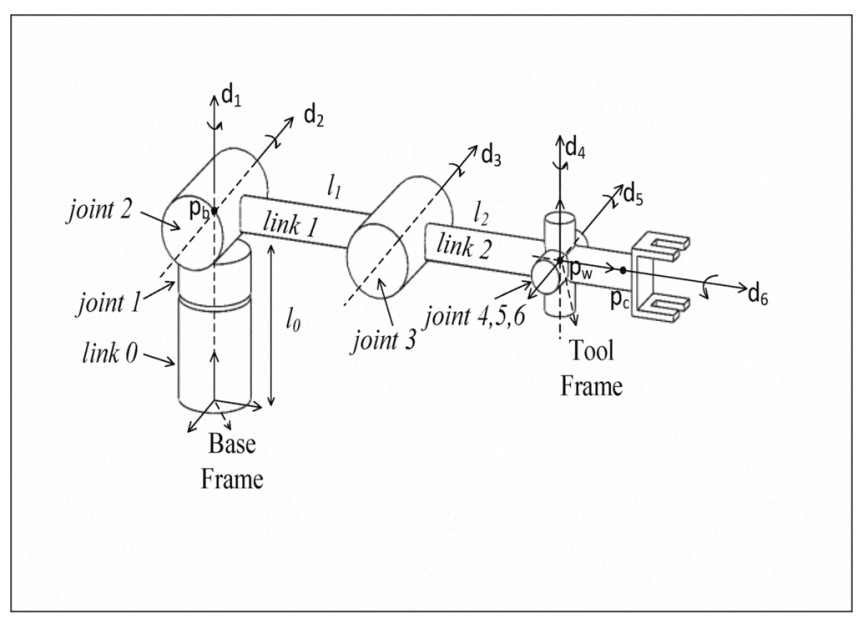

Figure 3: 6R Robot manipulator

\section{B. Inverse Kinematic}

We will use Paden - Kahan subproblems to obtain the inverse kinematic solution of serial robot manipulators. There are some Paden-Kahan subproblems and also new extended subproblems [16] [17] [18]. We will use just three of them which occur frequently in inverse solutions for common manipulator design. To solve the inverse kinematics problem, we reduce the full inverse kinematics problem into appropriate sub-problems. Here are some subproblems. [15]

1. Rotation about a single axis.

2. Rotation about two subsequent axes.

3. Rotation to a given distance
VII. 6R SERIAL Robot MaNiPUlator KinEMATIC MODEL

A. Forward Kinematic

First we must determine the axes for all joints. Axes can be chosen as follow

$d_{1}=\left[\begin{array}{lll}0 & 0 & 1\end{array}\right] \quad d_{2}=\left[\begin{array}{lll}0 & 1 & 0\end{array}\right] \quad d_{3}=\left[\begin{array}{lll}0 & 1 & 0\end{array}\right]$

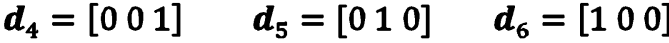

Any point on these axes can be written as:

$$
\begin{aligned}
& \boldsymbol{p}_{1}=\left[\begin{array}{lll}
0 & 0 & l_{0}
\end{array}\right] \\
& \boldsymbol{p}_{3}=\left[\begin{array}{lll}
l_{1} & 0 & l_{0}
\end{array}\right] \\
& p_{5}=\left[l_{1}+l_{2} 0 l_{0}\right] \quad p_{6}=\left[l_{1}+l_{2} 0 l_{0}\right]
\end{aligned}
$$

Thus we can write quaternions by using axes and point vectors. Quaternions can be obtained from equation (20) and (21) where $n=6$. To obtain translation, equation (21) must be calculated six times. Our general forward kinematic equation is given by

$\hat{q}_{16}=q_{1} \otimes q_{2} \otimes q_{3} \otimes q_{4} \otimes q_{5} \otimes q_{6}$ and

$\hat{q}_{i}^{o}=\hat{q}_{i-1} \otimes p_{i} \otimes \hat{q}_{i-1}^{*}-\hat{q}_{i} \otimes p_{i} \otimes \hat{q}_{i}^{*}+\hat{q}_{i-1}^{o}$

where $\mathrm{i}=1,2, \ldots 6$ and $\hat{q}_{1}^{o}=p_{1}-\hat{q}_{1} \otimes p_{1} \otimes \hat{q}_{1}^{*}$. And the position of the end effector is given by

$\hat{q}_{e p}=\hat{q}_{6} \otimes p_{e p} \otimes \hat{q}_{6}^{*}+\hat{q}_{6}^{o}$

\section{B. Inverse Kinematic}

In the inverse kinematic problem of the serial robot arm, we have orientation and position knowledge of the end effector. These are two quaternions and we will calculate all joint angles by using these quaternions. To find all joint angles complete inverse kinematic problem must be converted into the appropriate subproblems. First we put two points at the intersection of the axes. The first one is $\boldsymbol{p}_{\boldsymbol{w}}$ which is at the intersection of the wrist axes and the second one is $\boldsymbol{p}_{\boldsymbol{b}}$ which is at the intersection of the first two axes. The last three joints angles do not affect the point $\boldsymbol{p}_{\boldsymbol{w}}$. Hence we can say the position of point $\boldsymbol{p}_{\boldsymbol{w}}$ is free from the wrist angles. If we take the end effector position $q_{i n}^{0}=\left(q_{0}^{0}, q_{1}^{0}, q_{2}^{0}, q_{3}^{0}\right)$ we get $p_{w}=q_{i n}^{0}$. Thus we can write

$\hat{q}_{3} \otimes p_{w} \otimes \hat{q}_{3}^{*}+\hat{q}_{3}^{o}-p_{b}=q_{i n}^{0}-p_{b}$

Using the property that distance between points is preserved by rigid motions; take the magnitude of both sides of equation (27)

$\left\|\hat{q}_{3} \otimes p_{w} \otimes \hat{q}_{3}^{*}+\hat{q}_{3}^{o}-p_{b}\right\|=\left\|q_{i n}^{0}-p_{b}\right\|$

We obtain subproblem3. $\theta_{3}$ can be found by using subproblem 3 as

$\theta_{3}=\theta_{0} \pm \cos ^{-1}\left(\frac{\left\|\boldsymbol{u}^{\prime}||^{2}+|| v^{\prime}\right\|^{2}-{\delta^{\prime}}^{2}}{2|| \boldsymbol{u}^{\prime}|| \mid \boldsymbol{v}^{\prime} \|}\right)$ 


$$
\theta_{0}=\operatorname{atan} 2\left(d_{3}^{T}\left(u^{\prime} \times v^{\prime}\right), u^{\prime T} v^{\prime}\right)
$$

Here $u^{\prime}=\left(p_{w}-r\right)-d_{3} d_{3}^{T}\left(p_{w}-r\right)$

$$
\begin{gathered}
v^{\prime}=\left(p_{b}-r\right)-d_{3} d_{3}^{T}\left(p_{b}-r\right) \\
{\delta^{\prime}}^{2}=\left(\sqrt[2]{\left(q_{i n}^{0}-p b\right)\left(q_{i n}^{0}-p b\right)}\right)^{2}-\left|d_{3}^{T}\left(p_{w}-p_{b}\right)\right|^{2}
\end{gathered}
$$

where $\mathbf{r}$ is any point on the axis of $\boldsymbol{d}_{\mathbf{3}}$. Thus $\theta_{3}$ can be obtained. If we translate $p_{w}$ by using known $\theta_{3}$ we obtain a new point $p$. Here the point $p$ can be formulized as

$p=q_{3} \otimes p_{w} \otimes q_{3}^{*}+p_{b}-q_{3} \otimes p_{3} \otimes q_{3}^{*}$

For a known point $p$ we get subproblem 2 which can be expressed as

$$
\hat{q}_{3} \otimes p_{w} \otimes \hat{q}_{3}^{*}+\hat{q}_{3}^{o}-p_{b}=q_{\text {in }}^{0}
$$

Hence we can find $\theta_{1}$ and $\theta_{2}$ as follow:

$$
\theta_{2}=\operatorname{atan} 2\left(d_{2}^{T}\left(u^{\prime} \times v^{\prime}\right), \boldsymbol{u}^{\prime T} v^{\prime}\right)
$$

where

$$
\begin{aligned}
& u^{\prime}=(p-r)-d_{2} d_{2}^{T}(p-r) \\
& v^{\prime}=(v-r)-d_{2} d_{2}^{T}(v-r)
\end{aligned}
$$

where $v=\alpha d_{1}+\beta d_{2}+\gamma\left(d_{1} \times d_{2}\right)+r$

$$
\begin{gathered}
\alpha=\frac{\left(d_{1}^{T} d_{2}\right) d_{2}^{T}(p-r)-d_{1}^{T}\left(q_{i n}^{0}-r\right)}{\left(d_{1}^{T} d_{2}\right)^{2}-1} \\
\beta=\frac{\left(d_{1}^{T} d_{2}\right) d_{1}^{T}\left(q_{i n}^{0}-r\right)-d_{2}^{T}(p-r)}{\left(d_{1}^{T} d_{2}\right)^{2}-1} \\
\gamma^{2}=\frac{\|p-r\|^{2}-\alpha^{2}-\beta^{2}-2 \alpha \beta d_{1}^{T} d_{2}}{\left\|d_{1} \times d_{2}\right\|^{2}}\left(d_{1} \times d_{2}\right)
\end{gathered}
$$

$\theta_{1}=\operatorname{atan} 2\left(-d_{1}^{T}\left(u^{\prime} \times v^{\prime}\right), u^{\prime T} v^{\prime}\right)$

where $u^{\prime}=\left(q_{\text {in }}^{0}-r\right)-d_{1} d_{1}^{T}\left(q_{\text {in }}^{0}-r\right)$

$$
v^{\prime}=(v-r)-d_{1} d_{1}^{T}(v-r)
$$

where $\boldsymbol{v}$ is same as equation (35) and $\boldsymbol{r}$ is the intersection point of the axis one and axis two.

To find wrist angles we put a point $\boldsymbol{p}_{\boldsymbol{c}}$ which is on the $\boldsymbol{d}_{6}$ axis and it does not intersect with $\boldsymbol{d}_{\mathbf{4}}$ and $\boldsymbol{d}_{\mathbf{5}}$ axes. Thus the point $\boldsymbol{p}_{\boldsymbol{c}}$ is not affected from the last joint angle. Fourth and fifth joints angles determine the position of the point $\boldsymbol{p}_{\boldsymbol{c}}$. For known $\theta_{1}, \theta_{2}$ and $\theta_{3}$ we can write

$$
\begin{aligned}
q_{4-5} \otimes p_{c} \otimes q_{4-5}^{*}+ & \hat{q}_{3}^{*} \otimes \hat{q}_{4-6}^{o} \otimes \hat{q}_{3} \\
& =\hat{q}_{3}^{*} \otimes \hat{q}_{i n}^{o} \otimes \hat{q}_{3}-\hat{q}_{3}^{*} \otimes \hat{q}_{3}^{o} \otimes \hat{q}_{3}
\end{aligned}
$$

Equation (39) gives us subproblem2. $\theta_{4}$ and $\theta_{5}$ can be found as follow:

$$
\theta_{5}=\operatorname{atan} 2\left(d_{5}^{T}\left(u^{\prime} \times v^{\prime}\right), u^{\prime T} v^{\prime}\right)
$$

where

$$
\begin{aligned}
& u^{\prime}=\left(p_{g}-r\right)-d_{5} d_{5}^{T}\left(p_{g}-r\right) \\
& v^{\prime}=(v-r)-d_{5} d_{5}^{T}(v-r)
\end{aligned}
$$

where

$$
v=\alpha d_{4}+\beta d_{5}+\gamma\left(d_{4} \times d_{5}\right)+r
$$

$$
\alpha=\frac{\left(d_{4}^{T} d_{5}\right) d_{5}^{T}\left(p_{g}-r\right)-d_{4}^{T}\left(p_{c}-r\right)}{\left(d_{4}^{T} d_{5}\right)^{2}-1}
$$

$$
\beta=\frac{\left(d_{4}^{T} d_{5}\right) d_{4}^{T}\left(p_{c}-r\right)-d_{5}^{T}\left(p_{g}-r\right)}{\left(d_{4}^{T} d_{5}\right)^{2}-1}
$$

$$
\gamma^{2}=\frac{\left\|p_{g}-r\right\|^{2}-\alpha^{2}-\beta^{2}-2 \alpha \beta d_{4}^{T} d_{5}}{\left\|d_{4} \times d_{5}\right\|^{2}}\left(d_{4} \times d_{5}\right)
$$

where $\quad p_{g}=q_{m}^{*} \otimes p_{c} \otimes q_{m}+q_{m}^{*} \otimes p_{0} \otimes q_{m}+q_{m}^{*} \otimes p_{1} \otimes q_{m}-$ $q_{t 1}-q_{t 2}$

$$
\begin{aligned}
& \text { Here } \begin{aligned}
q_{m} & =\left(q_{1} \otimes q_{2} \otimes q_{3}\right)^{*} \otimes q_{i n}, \\
q_{t 1}= & q_{3}^{*} \otimes p_{3} \otimes q_{3}-p_{3} \\
q_{t 2}= & q_{3}^{*} \otimes p_{2} \otimes q_{3}-\left(q_{2} \otimes q_{3}\right)^{*} \otimes p_{3} \otimes\left(q_{2} \otimes q_{3}\right) \\
& +\left(q_{2} \otimes q_{3}\right)^{*} \otimes p_{1} \otimes\left(q_{2} \otimes q_{3}\right)
\end{aligned} \\
& \theta_{4}=\operatorname{atan} 2\left(-\boldsymbol{d}_{4}^{T}\left(\boldsymbol{u}^{\prime} \times \boldsymbol{v}^{\prime}\right), \boldsymbol{u}^{\prime T} \boldsymbol{v}^{\prime}\right)
\end{aligned}
$$

where

$$
\begin{aligned}
& u^{\prime}=\left(p_{c}-r\right)-d_{4} d_{4}^{T}\left(p_{c}-r\right) \\
& v^{\prime}=(v-r)-d_{4} d_{4}^{T}(v-r)
\end{aligned}
$$

where $v$ is same as equation (42) and $\boldsymbol{r}$ is the intersection point of the wrist axes. Thus first five joints angles are obtained. Only the last joint angle is unknown. The last joint angle can be found from orientation part of input. We can write,

$q_{16}=q_{1} \otimes q_{2} \otimes q_{3} \otimes q_{4} \otimes q_{5} \otimes q_{6}=q_{\text {in }}$

$q_{6}=\left(q_{1} \otimes q_{2} \otimes q_{3} \otimes q_{4} \otimes q_{5}\right)^{*} \otimes q_{i n}$

We can find the last joint angle from equation (46).

\section{SimUlation RESUlTS}

6R-robot manipulator forward and inverse kinematic problems are solved by using presented method and also D-H convention. Simulation results are obtained by using Matlab. These two methods are compared in terms of computation 
efficiency, singularity avoiding and accuracy. Some simulation results are given below. As we can see from table II and table III screw theory is a singularity avoiding method and it is more accurate than D-H convention. In singular case, however we can find finite inverse kinematic solutions when we use screw theory, we can't find finite (or real) solutions when we use D-H convention. Screw theory solutions errors are smaller than D-H convention solutions and also its solutions can be obtained faster than D-H convention. Computation efficiency can be observed from figure 4 . Running environment is as table I.

Table I: Running Environment

\begin{tabular}{|l|l|}
\hline CPU & Intel Core 2 Duo 2.2 GHz \\
\hline CPU MEMORY & $2 \mathrm{~GB}$ \\
\hline OPERATING SYSTEM & Windows XP \\
\hline SIMULATION SOFTWARE & MATLAB 7 \\
\hline
\end{tabular}

Table II: Nonsingular Case:

\begin{tabular}{|l|l|l|l|l|}
\hline $\begin{array}{l}\text { Real } \\
\text { Angle }\end{array}$ & $\begin{array}{l}\text { Screw } \\
\text { Solutions }\end{array}$ & $\begin{array}{l}\text { Screw } \\
\text { Error }\end{array}$ & $\begin{array}{l}\text { D-H } \\
\text { Solutions }\end{array}$ & $\begin{array}{l}\text { D-H } \\
\text { Error }\end{array}$ \\
\hline$\theta 1=0.6283$ & $\theta 1=0.6283$ & 0 & $\theta 1=0.6283$ & 0 \\
\hline$\theta 2=0.5236$ & $\theta 2=0.5236$ & 0 & $\theta 2=0.5236$ & 0 \\
\hline$\theta 3=0.4488$ & $\theta 3=0.4488$ & 0 & $\theta 3=0.4488$ & 0 \\
\hline$\theta 4=0.5236$ & $\theta 4=0.5237$ & 0.0001 & $\theta 4=0.5255$ & 0.0019 \\
\hline$\theta 5=0.2856$ & $\theta 5=0.2856$ & 0 & $\theta 5=0.2855$ & 0.0001 \\
\hline$\theta 6=1.0472$ & $\theta 6=1.0471$ & 0.0001 & $\theta 6=1.0474$ & 0.0001 \\
\hline
\end{tabular}

Table III: Singular Case (Elbow \& Wrist Singularities):

\begin{tabular}{|l|l|l|}
\hline Real Angle & Quaternion & D-H Solutions \\
\hline$\theta 1=0.6283$ & $\theta 1=0.6283$ & Unreal \\
\hline$\theta 2=0.5236$ & $\theta 2=0.5236$ & Unreal \\
\hline$\theta 3=0$ & $\theta 3=0$ & Unreal \\
\hline$\theta 4=0.5236$ & $\theta 4=0.9282$ & Unreal \\
\hline$\theta 5=1.5708$ & $\theta 5=1.5614$ & Unreal \\
\hline$\theta 6=1.0472$ & $\theta 6=1.4579$ & Unreal \\
\hline
\end{tabular}

Note: In singular case some solutions are not same as real angle, because there are infinite solutions in singular case and one solution is chosen from infinite solutions.

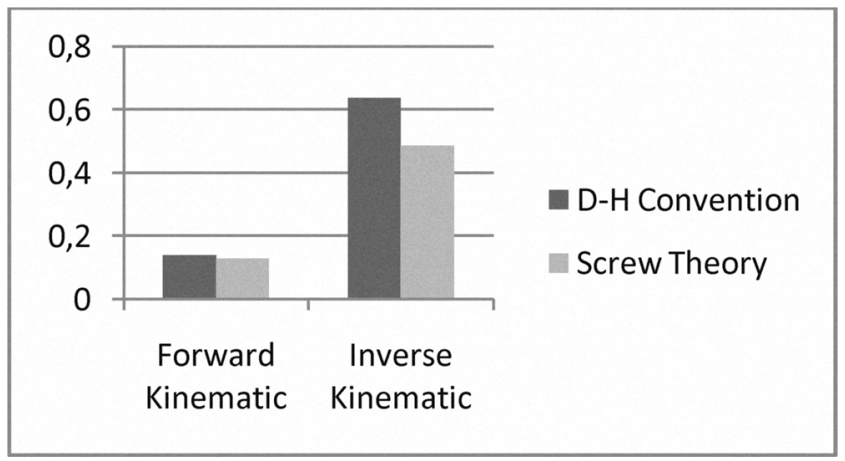

Figure4: Computation time of forward and inverse kinematic

\section{CONCLUSION}

We presented a new inverse kinematic solution by using screw theory. In this method quaternion is used as a screw operator. And also screw theory and homogeneous transformation approaches are investigated and compared with respect to singularity, computation efficiency and accuracy. Screw theory is a singularity avoiding method but homogeneous transformation is not. And also screw theory is more computationally efficient and more accurate than homogeneous transformation. Nevertheless homogenous transformations with D-H convention applications are more common than screw theory. Because point transformation can be understood easier than line transformation, its mathematical substructure is simpler than screw theory and also it is well defined method.

\section{REFERENCES}

[1] Mark W. Spong, Seth Hutchinson, M.Vidyasagar "Robot Modeling and Control" Jhon Wiley \& Sons 2006

[2] J.-H. Kim and V. R. Kumar, "Kinematics of robot manipulators via line transformations," J. Robot. Syst., vol. 7, no. 4, pp. 649-674, 1990.

[3] D. I. Pieper, "The kinematics of manipulators under computer control,"Tech. Rep., Stanford Artif. Intell. Lab., Stanford Univ., Stanford, CA,AIM 72, 1968.

[4] E. A. Maxwell, General Homogeneous Coordinates in Space of ThreeDimensions. Cambridge, U.K.: Cambridge Univ. Press, 1951.

[5] R.S. Ball,"The Theory of Screw" Cambridge Univ. Press, 1951

[6] Z. Huandg and Y.L. Yao,"Extension of Usable Workspace of Rotational Axes in Robot Planning", Robotica (1999) volume 17, pp. 293-301 Printed in the United Kingdom (C) 1999 Cambridge University Press

[7] W. R. Hamilton, Elements of Quaternions, Vol. I \& II. New York: Chelsea, 1869

[8] J. Funda and R. P. Paul, "A computational analysis of screw transformations in robotics," IEEE Trans. Robot. Automat., vol. 6, pp. 348-356,June 1990

[9] J.Funda. R.H. Taylor and R. P. Paul, "On homogeneous transforms quaternions, and computational efficiency," IEEE Trans. Robot. Automat. Vol 6, pp 382-388 June 1990

[10] A.T. Yang and F. Freudenstein, "Application of dual-number quaternion algebra to the analysis of spatial mechanisms," ASME J Appl. Mech. E,vol. 31, no. 2, pp 300-308, June 1964

[11] Jack C. K. Chou "Quaternion Kinematic and Dynamic Differential Equations", IEEE Transactions on Robotics and Automation VOL. 8 No. 1, February 1992

[12] Qing Tan; Balchen, J.G. "General quaternion transformation representation for roboticapplication", International Conference on Volume, Issue , 17-20 Oct 1993 Page(s):319 - 324 vol.3

[13] H. Bruyninckx and J. D. Schutter, "Introduction toIntelligent Robotics" 7th edition 1October 2001

[14] R. Mukundan , "Quaternions: From Classical Mechanics to Computer Graphics, and Beyond" , Proceedings of the 7th Asian Technology Confrence in Mathematics 2002

[15] J. M. Selig, "Geometrical Foundation Of Robotics", World Scientific Publishing 2000

[16] M.Murray, Z.Li and S.S. Sastry, "A mathematical introduction to robotic manipulation," Boca Raton FL:CRC Press, 1994

[17] J.Xie,W.Qiang ,B.Liang and C.Li "Inverse Kinematics Problem for 6DOF Sapce Manipulator Based On The Theory of Screw", International Conference on Robotics and Biomimetics, Dec. 2007

[18] T. Yue-sheng , X. Ai-ping "Extension of the Second Paden-Kahan Sub-problem and its' Application in the Inverse Kinematics of a Manipulator”, 978-1-4244-1676-9/08 (C2008 IEEE) 